\title{
Generating normal numbers over Gaussian integers
}

\author{
by \\ MANFRed G. Madritsch (Graz)
}

1. Introduction. When considering number systems, one is especially interested in uniqueness, periodicity and randomness of representations. In this paper we deal with the last property, in particular, we are concerned with the distribution of blocks in an expansion. We will call a number normal in a number system if every possible block of finite size occurs asymptotically with the same frequency.

For number systems over the reals this has been studied for a very long time. The quantitative aspect is that almost every real number is normal with respect to the Lebesgue measure. But we still do not know whether $\pi$ or $\log 2$ is normal in a given base $q \geq 2$.

On the other hand, we know how to construct normal numbers. This started with the construction of Champernowne who was able to show that

$$
0.123456789101112 \ldots
$$

is normal in base 10. This idea was successively extended to the integer part of polynomials over the positive integers by Davenport and Erdös [3] (polynomials with integer coefficients), Schiffer [24] (polynomials with rational coefficients), and Nakai and Shiokawa [22] (polynomials with real coefficients). Finally, it was shown by Madritsch et al. [20] that

$$
0 .\lfloor f(1)\rfloor\lfloor f(2)\rfloor\lfloor f(3)\rfloor\lfloor f(4)\rfloor\lfloor f(5)\rfloor\lfloor f(6)\rfloor \ldots
$$

is normal if $f$ is an entire function of bounded logarithmic order and $\lfloor x\rfloor$ denotes the expansion of the integer part of $x$ with respect to a given base $q \geq 2$.

In this paper our aim is to generalize the above mentioned construction of normal numbers to number systems for Gaussian integers. The properties

2000 Mathematics Subject Classification: 11K16, 11A63.

Key words and phrases: normal numbers, Gaussian integers, canonical number system.

Supported by the Austrian Research Foundation (FWF), Project S9611, that is part of the Austrian Research Network "Analytic Combinatorics and Probabilistic Number Theory". 
of these number systems have been investigated for instance by Kátai and Szabó [14] as well as Grabner et al. [7].

2. Definitions of number systems and normality. We start by recalling some well-known definitions pertaining to number systems, mainly following [6].

Let $b \in \mathbb{Z}[i]$ and let $\mathcal{D}$ be a complete set of residue classes modulo $b$. Then we call $(b, \mathcal{D})$ a number system (NS) if every $z \in \mathbb{Z}[i]$ has a unique and finite representation

$$
z=\sum_{k=0}^{\ell-1} d_{k}(z) b^{k},
$$

where $d_{k}(z)=0$ for $k \geq \ell$. We call $d_{k}(z) \in \mathcal{D}$ the digits of $z$.

Furthermore, $b$ is called the base and $\mathcal{D}$ the set of digits of the NS. We let $\ell(z):=\max \left\{k: d_{k-1}(z) \neq 0\right\}$ denote the length of the expansion. If $\mathcal{D}=\{0,1, \ldots,|N(b)|-1\}$ where $N(b)$ denotes the norm of $b$ over $\mathbb{Q}$ then we call $(b, \mathcal{D})$ a canonical number system (CNS).

One of the first who considered the possible bases for a CNS was Knuth [15], who was able to show that $b=-1 \pm i$ is a base. Later this was generalized by Kátai and Szabó [14] who proved that $b=-n \pm i$ with $n \in \mathbb{N}$ are all possible bases for the Gaussian integers. This was further generalized to algebraic number fields and matrix number systems in a series of papers (see for instance $[1,8,12,13,17,18,21,23]$ ).

In order to define uniform distribution and normal numbers we need an equivalent for the "reals". Therefore we will extend our number system onto $\mathbb{C}$. By Theorem 2 in [14], every $\gamma \in \mathbb{C}$ has a (not necessarily unique) representation of the shape

$$
\gamma=\sum_{k=-\infty}^{\ell(\gamma)} d_{k}(\gamma) b^{k} \quad\left(d_{k}(\gamma) \in \mathcal{D}\right) .
$$

In this context the fundamental domain $\mathcal{F}^{\prime}$ indicates the properties of this extension. It is defined as all numbers with zero in the integer part of their $b$-ary representation, i.e.,

$$
\mathcal{F}^{\prime}:=\left\{\gamma \in \mathbb{C} \mid \gamma=\sum_{k \geq 1} d_{k} b^{-k}, d_{k} \in \mathcal{D}\right\} .
$$

It is known (see $[8,10])$ that

$$
\bigcup_{a \in \mathbb{Z}[i]}\left(\mathcal{F}^{\prime}+a\right)=\mathbb{C}
$$


and

$$
\lambda\left(\left(\mathcal{F}^{\prime}+a\right) \cap\left(\mathcal{F}^{\prime}+b\right)\right)=0 \quad(a, b \in \mathbb{Z}[i], a \neq b),
$$

where $\lambda$ denotes the Lebesgue measure on $\mathbb{C}$.

Then we denote by

$$
\lfloor\gamma\rfloor_{b}=\lfloor\gamma\rfloor:=\sum_{k=0}^{\ell(\gamma)} d_{k}(\gamma) b^{k}
$$

the integer part with respect to base $b$. This definition may be ambiguous, but in view of (2.3), the integer part is well-defined for almost all Gaussian integers, which suffices for our purpose (we note that one can avoid the ambiguity by choosing always the greatest or least integer with respect to the ordering defined below).

We fix a base $b$ and turn to the definition of normal numbers. Let $d_{1} \ldots d_{l} \in \mathcal{D}^{l}$ be a block of digits of length $l$. We denote by $\mathcal{N}\left(\theta ; d_{1} \ldots d_{l} ; N\right)$ the number of occurrences of this block in the first $N$ digits of $\theta$. Thus

$$
\mathcal{N}\left(\theta ; d_{1} \ldots d_{l} ; N\right):=\#\left\{1 \leq n \leq N: d_{1}=d_{n}(\theta), \ldots, d_{l}=d_{n+l-1}(\theta)\right\} .
$$

Now we call $\theta$ normal in $(b, \mathcal{D})$ if for every $l \geq 1$ we have

$$
\mathcal{R}_{N}(\theta)=\mathcal{R}_{N, l}(\theta):=\sup _{d_{1} \ldots d_{l}}\left|\frac{1}{N} \mathcal{N}\left(\theta ; d_{1} \ldots d_{l} ; N\right)-\frac{1}{|\mathcal{D}|^{l}}\right|=o(1)
$$

where the supremum is taken over all blocks of length $l$.

By our completion of $\mathbb{Q}(i)$ to $\mathbb{C}$ we know that there can be more than one representation for a $\gamma \in \mathbb{C}($ see $[10,11,21])$. We call a $\gamma \in \mathbb{C}$ ambiguous if

$$
\gamma=\sum_{k=-\infty}^{\ell(\gamma)} x_{k} b^{k}=\sum_{k=-\infty}^{\ell(\gamma)} y_{k} b^{k}
$$

with $x_{k} \neq y_{k}$ for at least one $k \leq \ell(\gamma)$. These numbers are dealt with in the following lemma.

Lemma 2.1 ([19, Proposition] $]$. Let $(b, \mathcal{D})$ be a CNS. Then no number with an ambiguous representation is normal.

As we want to construct a normal number as a concatenation of digital expansions of a certain sequence of numbers we have to give an ordering for the Gaussian integers which will fit our purpose. We set $q:=N(b)$ where $N$ denotes the norm of $b$ over $\mathbb{Q}$ and let $\tau$ be a bijection between $\mathcal{D}$ and $\{0,1, \ldots, q-1\}$ with $\tau(0)=0$. Then we extend $\tau$ to the Gaussian integers by setting $\tau\left(d_{0}+d_{1} b+d_{2} b^{2}+\cdots+d_{k} b^{k}\right):=\tau\left(d_{0}\right)+\tau\left(d_{1}\right) q+\tau\left(d_{2}\right) q^{2}+\cdots+\tau\left(d_{k}\right) q^{k}$. This is essentially a bijection because of the uniqueness of the representation of every Gaussian integer, i.e., $\tau(\mathbb{Z}[i])=\mathbb{N} \cup\{0\}=\mathbb{N}_{0}$. Thus for every 
$a \in \mathbb{Z}[i]$ there exists an $n \in \mathbb{N}_{0}$ such that $\tau(a)=n$. Now we pull back the relation $\leq$ from $\mathbb{N}_{0}$ to $\mathbb{Z}[i]$ by

$$
a \leq b: \Leftrightarrow \tau(a) \leq \tau(b), \quad a, b \in \mathbb{Z}[i] .
$$

Then the formula $z_{n}:=\tau^{-1}(n-1)$ defines an increasing sequence of elements of $\mathbb{Z}[i]$.

For a function $f: \mathbb{Z}[i] \rightarrow \mathbb{C}$ we define

$$
\theta_{b}(f):=\theta(f)=\left\lfloor f\left(z_{1}\right)\right\rfloor q^{-\ell\left(f\left(z_{1}\right)\right)}+\left\lfloor f\left(z_{2}\right)\right\rfloor q^{-\ell\left(f\left(z_{1}\right)\right)-\ell\left(f\left(z_{2}\right)\right)}+\cdots .
$$

This is simply the concatenation of the integer parts of the function values evaluated on the sequence $\left\{z_{n}\right\}_{n \geq 1}$ of Gaussian integers. We are now in a position to state our main theorem.

ThEOREM 2.2. Let $f(z)=\alpha_{d} z^{d}+\cdots+\alpha_{1} z+\alpha_{0}$ be a polynomial with coefficients in $\mathbb{C}$. Let $(b, \mathcal{D})$ be a CNS in the Gaussian integers. Then for every $l \geq 1$,

$$
\sup _{d_{1} \ldots d_{l}}\left|\frac{1}{N} \mathcal{N}\left(\theta_{b}(f) ; d_{1} \ldots d_{l} ; N\right)-\frac{1}{|\mathcal{D}|^{l}}\right| \ll(\log N)^{-1},
$$

where the supremum is taken over all blocks of length $l$.

3. Preliminary lemmas. The first lemma will help us to rewrite the asymptotics.

Lemma 3.1 ([20, Lemma 3.4]). Let $\left\{a_{n}\right\}_{n \geq 1}$ and $\left\{b_{n}\right\}_{n \geq 1}$ be two sequences of reals with $0<a_{n} \leq b_{n}$ for all $n$. Suppose that $\lim _{n \rightarrow \infty} a_{n} \neq 0$ and

$$
\lim _{n \rightarrow \infty} \frac{a_{n}}{b_{n}}=0 .
$$

Then

$$
\lim _{n \rightarrow \infty} \frac{\sum_{i=1}^{n} a_{i}}{\sum_{i=1}^{n} b_{i}}=0
$$

As we deal with blocks of a certain length we need information about the connection of the norm of a Gaussian integer and the length of its expansion. This connection is described by the following lemma.

Lemma 3.2 ([7, Proposition 2.6]). Let $(b, \mathcal{D})$ be a number system in the Gaussian integers and $q:=N(b)$. Then

$$
\left.\left|\ell(z)-\log _{q}\right| z\right|^{2} \mid \leq c_{b}
$$

for a constant $c_{b}$ depending only on the base $b$, where $\log _{q}$ is the logarithm in base $q$.

In the proof of our main result we will need the discrepancy (see [5, p. 5] for a definition) $D_{N}\left(\mathbf{x}_{n}\right)$ of the first $N$ elements of a sequence $\left\{\mathbf{x}_{n}\right\}_{n \geq 1}$ of elements in $\mathbb{R}^{2}$. The following result provides an estimate of the discrepancy. 
Lemma 3.3 (Erdős-Turán-Koksma inequality, [5, Theorem 1.21]). Let $\mathbf{x}_{1}, \ldots, \mathbf{x}_{N}$ be points in $\mathbb{R}^{2}$ and $T$ an arbitrary positive integer. Then

$$
D_{N}\left(\mathbf{x}_{n}\right) \leq\left(\frac{3}{2}\right)^{k}\left(\frac{2}{V+1}+\sum_{0<\|\mathbf{v}\|_{\infty} \leq V} \frac{1}{r(\mathbf{v})}\left|\frac{1}{N} \sum_{n=1}^{N} e\left(\mathbf{v} \cdot \mathbf{x}_{n}\right)\right|\right),
$$

where $r(\mathbf{v})=\left(\max \left\{1,\left|v_{1}\right|\right\}\right) \cdot\left(\max \left\{1,\left|v_{2}\right|\right\}\right)$ for $\mathbf{v}=\left(v_{1}, v_{2}\right) \in \mathbb{Z}^{2}$.

For the transformation of an exponential sum into an integral we will apply the following two lemmas.

Lemma 3.4 ([2, Lemma 5.4]). Suppose that $F\left(x_{1}, \ldots, x_{r}\right)$ is a real differentiable function for $0 \leq x_{j} \leq P_{j} \leq P(j=1, \ldots, r)$, the function $\partial F\left(x_{1}, \ldots, x_{r}\right) / \partial x_{j}$ is piecewise monotone and of constant sign in each $x_{j}$ $(j=1, \ldots, r)$ for any fixed values of the other variables, and the number of the intervals of monotonicity and constant sign does not exceed s. Moreover, suppose that

$$
\left|\frac{\partial F\left(x_{1}, \ldots, x_{r}\right)}{\partial x_{j}}\right| \leq \delta, \quad j=1, \ldots, r
$$

for some $0<\delta<1$. Then

$$
\begin{aligned}
\sum_{x_{1}=0}^{P_{1}} & \ldots \sum_{x_{r}=0}^{P_{r}} e\left(F\left(x_{1}, \ldots, x_{r}\right)\right) \\
& =\int_{0}^{P_{1}} \cdots \int_{0}^{P_{r}} e\left(F\left(x_{1}, \ldots, x_{r}\right)\right) d x_{1} \ldots d x_{r}+\theta_{1} r s P^{r-1}\left(3+\frac{2 \delta}{1-\delta}\right)
\end{aligned}
$$

for some $\theta_{1}$ with $\left|\theta_{1}\right| \leq 1$.

Lemma 3.5 ([25, Lemma 4.2]). Let $F(x)$ be a real differentiable function such that $F^{\prime}(x)$ is monotonic, and $F^{\prime}(x) \geq m>0$, or $F^{\prime}(x) \leq-m<0$, throughout the interval $[a, b]$. Then

$$
\left|\int_{a}^{b} e(F(x)) d x\right| \leq \frac{4}{m}
$$

In the next lemma we give an application of the preceding ones.

Lemma 3.6. Let $M$ and $N$ be positive integers with $M \ll N$. Let $F$ : $\mathbb{C} \rightarrow \mathbb{C}$ satisfy the conditions of Lemmas 3.4 and 3.5. Then

$$
\sum_{M \leq|z|^{2}<M+N} e(\operatorname{tr}(F(z))) \ll \frac{\sqrt{N}}{m}+\frac{N}{(\log N)^{\sigma / 2}}+s \frac{3-\delta}{1-\delta} \sqrt{N(\log N)^{\sigma}}
$$

for any positive real number $\sigma$. Here $\operatorname{tr}(x)$ denotes the trace of an element $x \in \mathbb{Z}[i]$. 
Proof. This is a generalization of [6, Lemmas 2.1 and 2.2]. In order to apply the two lemmas above we start by considering squares in the annulus $M \leq|z|^{2}<M+N$. Set $D_{\nu}:=\{z=x+i y \in \mathbb{Z}[i]:-\nu \leq x, y \leq \nu\}$. By Lemma 3.4,

$$
\begin{aligned}
\sum_{z \in D_{\nu}} e(\operatorname{tr}(F(z))) & =\sum_{x=-\nu}^{\nu} \sum_{y=-\nu}^{\nu} e(\operatorname{tr}(F(x+i y))) \\
& =\int_{-\nu}^{\nu} \int_{-\nu}^{\nu} e(\operatorname{tr}(F(x+i y))) d x d y+2 \theta_{1} s \nu \frac{3-\delta}{1-\delta} .
\end{aligned}
$$

We take the modulus in order to apply Lemma 3.5:

$$
\begin{aligned}
\left|\sum_{z \in D_{\nu}} e(\operatorname{tr}(F(z)))\right| & \leq \int_{-\nu}^{\nu}\left|\int_{-\nu}^{\nu} e(\operatorname{tr}(F(x+i y))) d x\right| d y+2 \theta_{1} s \nu \frac{3-\delta}{1-\delta} \\
& \leq 2 \nu \max _{-\nu \leq y \leq \nu}\left|\int_{-\nu}^{\nu} e(\operatorname{tr}(F(x+i y))) d x\right|+2 \theta_{1} s \nu \frac{3-\delta}{1-\delta} \\
& \leq \frac{8 \nu}{m}+2 \theta_{1} s \nu \frac{3-\delta}{1-\delta} .
\end{aligned}
$$

Secondly we tessellate the annulus $M \leq|z|^{2}<M+N$ by squares of side length $\sqrt{N /(\log N)^{\sigma}}$. We define two sets $I$ and $B$ to consist of the squares which are completely inside the annulus and those which intersect the boundary, respectively. Let $C_{I}$ and $C_{B}$ be their respective contributions to the sum. There are $\mathcal{O}\left((\log N)^{\sigma}\right)$ squares in $I$ and together with our considerations above we get

$$
C_{I} \ll \frac{N}{m}+s \frac{3-\delta}{1-\delta} \sqrt{N(\log N)^{\sigma}} .
$$

For the boundary we see that there are two annuli of width $\mathcal{O}\left(\sqrt{M /(\log M)^{\sigma}}\right)$ and $\mathcal{O}\left(\sqrt{(M+N) /(\log M+N)^{\sigma}}\right)$ that cover the boundary. By noting that $M \ll N$ we get

$$
C_{B} \ll \frac{N}{(\log N)^{\sigma / 2}} .
$$

This together with the estimation above yields the result.

Finally, we need an estimation for a complete exponential sum in the Gaussian rationals.

Lemma 3.7 ([9, Theorem 1]). Let $f$ be a kth degree polynomial with coefficients in $\mathbb{Q}(i)$ and $q$ be the least common multiple of its coefficients. If $\Lambda(q)$ is a complete set of residues modulo $q$, then, for any $\varepsilon>0$,

$$
\sum_{\lambda \in \Lambda(q)} e(\operatorname{tr}(f(\lambda))) \ll(N(q))^{1-1 / k+\varepsilon},
$$

where the implied constant depends only on $f$ and $\varepsilon$. 
4. Properties of the fundamental domain. In this section we mainly follow the paper of Gittenberger and Thuswaldner [6]. Let $b=-n+i$ be a base of a CNS in $\mathbb{Z}[i]$. Then every $\gamma \in \mathbb{C}$ has a unique representation of the shape $\gamma=\alpha+\beta b$ with $\alpha, \beta \in \mathbb{R}$. Thus we define the mapping

$$
\varphi: \mathbb{C} \rightarrow \mathbb{R}^{2}, \quad \alpha+\beta b \mapsto(\alpha, \beta) .
$$

Since $(1, b)$ is an integral basis we know that $\varphi(\mathbb{Z}[i])=\mathbb{Z}^{2}$.

In order to ease things we use our embedding from above to switch from $\mathbb{C}$ to $\mathbb{R}^{2}$. Then we get the analog in $\mathbb{R}^{2}$ for the fundamental domain defined in $(2.2)$ :

$$
\mathcal{F}:=\varphi\left(\mathcal{F}^{\prime}\right)=\left\{\gamma \in \mathbb{R}^{2} \mid \gamma=\sum_{k \geq 1} d_{k} B^{-k}, d_{k} \in \varphi(\mathcal{D})\right\},
$$

where $B$ is the matrix corresponding to multiplication by $b$ in $\mathbb{R}^{2}$ given by

$$
B=\left(\begin{array}{cc}
0 & -1-n^{2} \\
1 & -2 n
\end{array}\right)
$$

We refer the reader to [23] for more details.

Now we define for every $a \in \mathbb{Z}[i]$ the domain corresponding to the elements of $\mathcal{F}$ whose digit representation after the comma starts with the digits of the expansion of $a$. In particular, we set

$$
\mathcal{F}_{a}=B^{-\ell(a)}(\mathcal{F}+\varphi(a)) .
$$

As in the case of normal numbers in the reals we need an Urysohn function for this fundamental domain of numbers starting with $a$. In the reals we use a lemma due to Vinogradov (cf. Lemma 2 of [26, p. 196]); in $\mathbb{C}$, however, we have to construct a corresponding version of this lemma.

For $a \in \mathcal{D}$ this has been done by Gittenberger and Thuswaldner in Section 3 of [6]. As the generalization of their construction to the case of $a \in \mathbb{Z}[i]$ runs along the same lines, we only state the corresponding results and leave their proofs to the reader.

Lemma 4.1 ([6, Lemma 3.1]). For all $a \in \mathbb{Z}[i]$ and all $k \in \mathbb{N}$ there exists an axis-parallel tube $P_{k, a}$ with the following properties:

(1) $\partial \mathcal{F}_{a} \subset P_{k, a}$ for all $k \in \mathbb{N}$,

(2) $\lambda_{2}\left(P_{k, a}\right)=\mathcal{O}\left(\mu^{k} /|b|^{2 k}\right)$,

(3) $P_{k, a}$ consists of $\mathcal{O}\left(\mu^{k}\right)$ axis-parallel rectangles with $1<\mu<|b|^{2}$, each of which has Lebesgue measure $\mathcal{O}\left(|b|^{-2 k}\right)$.

Here we denote by $\lambda_{2}$ the usual Lebesgue measure on $\mathbb{R}^{2}$.

Gittenberger and Thuswaldner [6] define, for every pair $(k, a)$, suitable axis-parallel polygons $\Pi_{k, a}$. Then they show that $d\left(\Pi_{k, a}, \partial \mathcal{F}_{a}\right)<c|b|^{-k}$ for 
a constant $c>0$, where $d(\cdot, \cdot)$ denotes the Hausdorff metric, and set

$$
P_{k, a}:=\left\{\left.z \in \mathbb{R}^{2}\left|\left\|z-\Pi_{k, a}\right\|_{\infty} \leq 2 c\right| b\right|^{-k}\right\} .
$$

As in [6] we denote by $I_{k, a}$ the interior of $\Pi_{k, a}$ and define

$$
f_{a}(x, y)=\frac{1}{\Delta^{2}} \int_{-\Delta / 2}^{\Delta / 2} \int_{-\Delta / 2}^{\Delta / 2} \psi_{a}(x+\bar{x}, y+\bar{y}) d \bar{x} d \bar{y}
$$

where

$$
\Delta:=2 c_{\Delta}|b|^{-k}
$$

with $c_{\Delta}>0$ a constant and

$$
\psi_{a}(x, y)= \begin{cases}1 & \text { if }(x, y) \in I_{k, a}, \\ 1 / 2 & \text { if }(x, y) \in \Pi_{k, a}, \\ 0 & \text { otherwise }\end{cases}
$$

Now $f_{a}$ is the desired Urysohn function for $\mathcal{F}_{a}$ in $\mathbb{R}^{2}$. The Fourier analysis of this function yields

Lemma 4.2 ([6, Lemma 3.2]). Let $f_{a}(x, y)=\sum_{m, n} C(m, n) e(m x+n y)$ be the Fourier expansion of $f_{a}$. Then

$$
C(m, n)= \begin{cases}|b|^{-2 \ell(a)}, & m=n=0 \\ \mu^{k} c(m) c(n), & \text { otherwise }\end{cases}
$$

where

$$
c(t) \ll \begin{cases}1, & t=0, \\ \min \left(|t|^{-1}, \Delta|t|^{-2}\right), & \text { otherwise. }\end{cases}
$$

As the proof of this lemma runs along the same lines as that of $[6$, Lemma 3.2] we omit it.

The coefficient $C(0,0)$ will correspond to the main term and all others contribute to the error term. One of our main tools will be Weyl sums which will be discussed in the next section.

5. The Weyl sum. Throughout this section we denote by $f$ a polynomial with coefficients in $\mathbb{C}$. Thus

$$
f(z)=\alpha_{d} z^{d}+\alpha_{d-1} z^{d-1}+\cdots+\alpha_{1} z .
$$

The following generalization of Lemma 2 of Nakai and Shiokawa [22] will play a crucial rôle in the proof of Theorem 2.2.

Proposition 5.1. Let $G>0$ and $N \geq 2$. Let $s$ be an integer with $1 \leq s \leq d$, let $H_{i}, K_{i}(i=s+1, \ldots, d)$ be any positive constants, and let $H_{s}^{*}, K_{s}^{*}$ be constants such that 


$$
\begin{aligned}
& H_{s}^{*} \geq 2^{3(s+2)}+2^{s+3}\left(G+\max _{s<i \leq d} H_{i}\right)+s \sum_{i=s+1}^{d} K_{i}, \\
& K_{s}^{*} \geq 2^{3(s+2)}+2^{s+3}\left(G+\max _{s<i \leq d} H_{i}\right)+2 s \sum_{i=s+1}^{d} K_{i} .
\end{aligned}
$$

Suppose that there are Gaussian integers $a_{i}$ and $q_{i}(s<i \leq d)$ such that

$$
1 \leq\left|q_{i}\right|^{2} \leq(\log N)^{K_{i}} \quad \text { and } \quad\left|\alpha_{i}-\frac{a_{i}}{q_{i}}\right| \leq \frac{(\log N)^{H_{i}}}{q_{i} N^{i / 2}}
$$

but there exist no Gaussian integers $a_{s}$ and $q_{s}$ with $\left(a_{s}, q_{s}\right)=1$ such that

$$
1 \leq\left|q_{s}\right|^{2} \leq(\log N)^{K_{s}^{*}} \quad \text { and } \quad\left|\alpha_{s}-\frac{a_{s}}{q_{s}}\right| \leq \frac{(\log N)^{H_{s}^{*}}}{q_{s} N^{s / 2}} .
$$

Then

$$
\left|\sum_{|z|^{2} \leq N} e(\operatorname{tr}(f(z)))\right| \ll N(\log N)^{-G} .
$$

To prove the proposition we need two lemmas. The first deals with approximation by Gaussian integers.

Lemma 5.2 ([4, Theorem 4.5]). Given any $z=x+i y \in \mathbb{C}$ and $N \in \mathbb{N}$, there exist Gaussian integers a and $q$ with $0<|q|^{2} \leq N$ such that

$$
\left|z-\frac{a}{q}\right|<\frac{2}{|q| \sqrt{N}}
$$

Furthermore we need a lemma which considers the case where $s=d$, the degree of the polynomial $f$, i.e., the leading coefficient is already well approximable.

Lemma $5.3([6$, Proposition 2.1]). Let $(h, q)=1$ and

$$
g(x)=\frac{h}{q} x^{d}+\alpha_{d-1} x^{d-1}+\cdots+\alpha_{1} x+\alpha_{0}
$$

where $(\log N)^{H} \leq|q|^{2} \leq N^{d}(\log N)^{-H}$. Then

$$
\left|\sum_{|z|^{2}<N} e(\operatorname{tr}(g(z)))\right| \ll N(\log N)^{-G} \quad \text { with } \quad H \geq 2^{d+2} G+2^{3(d+2)} .
$$

In order to apply Lemma 5.3 recursively, we need a tool to rewrite it.

LEMMA 5.4 ([16, Lemma 26]). Let $f_{1}$ and $f_{2}$ be functions defined on a finite set $M$. Then

$$
\sum_{x \in M} e\left(f_{1}(x)+f_{2}(x)\right)=\sum_{x \in M} e\left(f_{1}(x)\right)+2 \pi \theta \sum_{x \in M}\left|f_{2}(x)\right|
$$

for some $\theta$ with $|\theta| \leq 1$. 
Corollary 5.5. Let $g(x)=\alpha_{d} x^{d}+\alpha_{d-1} x^{d-1}+\cdots+\alpha_{1} x+\alpha_{0} \in \mathbb{C}[X]$. If there exist $h, q \in \mathbb{Z}[X]$ such that $(h, q)=1$ and

$$
\left|\alpha_{d}-\frac{h}{q}\right| \leq \frac{(\log N)^{H}}{|q| N^{d / 2}}
$$

with $(\log N)^{H} \leq|q| \leq N^{d / 2}(\log N)^{-H}$ and $H \geq 2^{d+1} G+2^{3(d+2)-1}$, then

$$
\left|\sum_{|z|^{2}<N} e(\operatorname{tr}(g(z)))\right| \ll N(\log N)^{-G} .
$$

Proof. This easily follows from

$$
\left|\alpha_{d}-\frac{h}{q}\right| \leq \frac{(\log N)^{H}}{|q| N^{d / 2}} \leq N^{-d / 2}
$$

together with Lemmas 5.4 and 5.3 and

$$
\begin{aligned}
\left|\sum_{|z|^{2}<N} e(\operatorname{tr}(g(z)))\right| \leq & \left|\sum_{|z|^{2}<N} e\left(\operatorname{tr}\left(\frac{h}{q} z^{d}+\alpha_{d-1} z^{d-1}+\cdots+\alpha_{0}\right)\right)\right| \\
& +\sum_{|z|^{2}<N}\left|\operatorname{tr}\left(\left(\alpha_{d}-\frac{h}{q}\right) z^{d}\right)\right| \\
\ll & N(\log N)^{-G}+N^{1 / 2} .
\end{aligned}
$$

Now we can start the proof of Proposition 5.1.

Proof of Proposition 5.1. This proof mainly follows the ideas of Nakai and Shiokawa in their proof of Lemma 2 in [22].

We consider the different possibilities for $s$. If $s=d$, there is nothing to prove, as this is exactly the case of Corollary 5.5.

Let $s<d$. We denote by $k$ the least common multiple of $q_{s+1}, \ldots, q_{d}$. We have $k \in \mathbb{Z}[i]$ because the Gaussian integers are a unique factorization domain. We denote by $Q$ the integer such that $|k|^{2} Q \leq N<|k|^{2}(Q+1)$. By our assumptions we have

$$
1 \leq|k|^{2} \leq(\log N)^{K} \quad \text { with } \quad K=\sum_{i=s+1}^{d} K_{i}
$$

and

$$
N(\log N)^{-K} \ll Q \ll N /|k|^{2} .
$$

Now, since $\mathbb{Z}[i]$ is a Euclidean domain, for every $s \in \mathbb{Z}[i]$ there exist unique $q, r \in \mathbb{Z}[i]$ with $|r|^{2}<|k|^{2}$ such that $s=q k+r$. Thus there exists a complete residue system $R$ modulo $k$ with

$$
R \subset\{z \in \mathbb{Z}[i]:|z| \leq|k|\} .
$$


We use this system to tessellate the open $\operatorname{disc} D:=\left\{z:|z|^{2}<N\right\}$ with translates of $R$. Let

$$
\begin{aligned}
T & :=\{t \in \mathbb{Z}[i]:(R+t k) \cap D \neq \emptyset\}, \\
I & :=\{t \in T: R+t k \subset D\} .
\end{aligned}
$$

As there are $\mathcal{O}(\sqrt{N})$ points on the circumference and $\mathcal{O}(|k|)$ points in $R$ we get

$$
\sum_{|z|^{2} \leq N} e(\operatorname{tr}(f(z)))=\sum_{t \in I} \sum_{r \in R} e(\operatorname{tr}(f(t k+r)))+\mathcal{O}(\sqrt{N}|k|) .
$$

As in the proof of Lemma 2 of Nakai and Shiokawa in [22] we want to perform Abel summation. Therefore we need an ordering on $I$. For $x, y \in I$, define

$$
x \prec y: \Leftrightarrow\left\{\begin{array}{l}
|x|<|y| \text { or } \\
(|x|=|y| \text { and } \arg (x)<\arg (y)) .
\end{array}\right.
$$

By the polar representation of every complex number, this ordering is well defined. Furthermore, we let $\sigma: \mathbb{N} \rightarrow I$ be a bijection such that $\sigma(1)=0$, $\sigma(|I|)=\max I$ (maximum with respect to $\prec$ ), and define

$$
\sigma(x) \prec \sigma(y): \Leftrightarrow x<y \text {. }
$$

Let $M=|I|$. Then

$$
\sum_{|z|^{2} \leq N} e(\operatorname{tr}(f(z)))=\sum_{n=1}^{M} \sum_{r \in R} e(\operatorname{tr}(f(\sigma(n) k+r)))+\mathcal{O}(\sqrt{N}|k|) .
$$

Now we are ready to perform Abel summation and define for short

$$
\begin{aligned}
\psi_{r}(x) & =\sum_{i=s+1}^{d} \gamma_{i}(x k+r)^{i}, & \gamma_{i} & =\alpha_{i}-\frac{a_{i}}{q_{i}}, \\
\varphi_{r}(x) & =\sum_{i=1}^{s} \alpha_{i}(x k+r)^{i}, & T_{r}(\ell) & =\sum_{n=1}^{\ell} e\left(\operatorname{tr}\left(\varphi_{r}(\sigma(n))\right)\right) .
\end{aligned}
$$

By the linearity of the trace tr we get

$$
\begin{aligned}
\sum_{n=1}^{M} \sum_{r \in R} e(\operatorname{tr}(f(\sigma(n) k+r))) & \\
= & \sum_{r \in R} \sum_{n=1}^{M} e\left(\operatorname{tr}\left(\sum_{i=1}^{d} \alpha_{i}(\sigma(n) k+r)^{i}\right)\right) \\
= & \sum_{r \in R} \sum_{n=1}^{M} e\left(\operatorname{tr}\left(\sum_{i=1}^{s} \alpha_{i}(\sigma(n) k+r)^{i}+\sum_{i=s+1}^{d} \alpha_{i}(\sigma(n) k+r)^{i}\right)\right)
\end{aligned}
$$




$$
\begin{aligned}
= & \sum_{r \in R} \sum_{n=1}^{M} e\left(\operatorname{tr}\left(\varphi_{r}(\sigma(n))+\sum_{i=s+1}^{d}\left(\gamma_{i}+\frac{a_{i}}{q_{i}}\right)(\sigma(n) k+r)^{i}\right)\right) \\
= & \sum_{r \in R} e\left(\operatorname{tr}\left(\sum_{i=s+1}^{d} \frac{a_{i}}{q_{i}} r^{i}\right)\right) \sum_{n=1}^{M} e\left(\operatorname{tr}\left(\varphi_{r}(\sigma(n))+\psi_{r}(\sigma(n))\right)\right) \\
= & \sum_{r \in R} e\left(\operatorname{tr}\left(\sum_{i=s+1}^{d} \frac{a_{i}}{q_{i}} r^{i}\right)\right) \sum_{n=1}^{M} e\left(\operatorname{tr}\left(\psi_{r}(\sigma(n))\right)\right)\left(T_{r}(n)-T_{r}(n-1)\right) \\
= & \sum_{r \in R} e\left(\operatorname{tr}\left(\sum_{i=s+1}^{d} \frac{a_{i}}{q_{i}} r^{i}\right)\right)\left[e\left(\operatorname{tr}\left(\psi_{r}(\sigma(M+1))\right)\right) T_{r}(M)\right. \\
& +\sum_{n=1}^{M}\left(e\left(\operatorname{tr}\left(\psi_{r}(\sigma(n))\right)\right)-e\left(\operatorname{tr}\left(\psi_{r}(\sigma(n+1))\right)\right) T_{r}(n)\right] \\
\ll & \sum_{r \in R}\left[\left|T_{r}(M)\right|+\sum_{n=1}^{M}\left|e\left(\operatorname{tr}\left(\psi_{r}(\sigma(n))\right)\right)-e\left(\operatorname{tr}\left(\psi_{r}(\sigma(n+1))\right)\right)\right|\left|T_{r}(n)\right|\right] .
\end{aligned}
$$

As the trace is a linear functional we get

$$
\frac{d}{d x} \operatorname{tr}(f(x))=\operatorname{tr}\left(\frac{d f}{d x}\right) .
$$

Noting that $\operatorname{tr}(a) \ll|a|$ for $a \in \mathbb{C}$ and $|\sigma(n)-\sigma(n+1)| \ll N^{1 / 2}$ for $1<n$ $\leq M$, we apply the mean-value theorem to get

$$
\left|e\left(\operatorname{tr}\left(\psi_{r}(\sigma(n))\right)\right)-e\left(\operatorname{tr}\left(\psi_{r}(\sigma(n+1))\right)\right)\right| \ll|k| \sum_{i=s+1}^{d}\left|\gamma_{i}\right| N^{i / 2-1} \ll|k| \frac{(\log N)^{H}}{N}
$$

where $H=\max \left\{H_{i}: i=1, \ldots, s\right\}$. Thus

$$
\sum_{n=1}^{M} \sum_{r \in R} e(\operatorname{tr}(f(\sigma(n) k+r))) \ll \sum_{r \in R}\left[\left|T_{r}(M)\right|+|k| \frac{(\log N)^{H}}{N} \sum_{n=1}^{M}\left|T_{r}(n)\right|\right] .
$$

If we can show that

$$
\left|T_{r}(n)\right| \ll \frac{N}{|k|(\log N)^{G+H}}
$$

then we are done. We may assume that

$$
n \gg \frac{N}{|k|(\log N)^{G+H}} \text {. }
$$


We split the estimation of $T_{r}(n)$ according to whether or not there exist $a$ and $q$ with $(a, q)=1$ such that

$$
(\log N)^{H^{\prime}} \leq|q|^{2} \leq N^{s}(\log N)^{-H^{\prime}}
$$

and

$$
\left|k^{s} \alpha_{s}-\frac{a}{q}\right| \leq|q|^{2}
$$

with $H^{\prime}=2^{3(s+2)}+2^{s+3}(G+H)+s K$.

- Suppose there exist such $a$ and $q$. Then by the definition of $H^{\prime}$ together with (5.6) we get

$$
(\log n)^{h^{\prime}} \leq|q|^{2} \leq n^{s}(\log n)^{-h^{\prime}},
$$

where $h^{\prime}=2^{3(s+2)}+2^{s+2}(G+H)$. Thus an application of Lemma 5.3 yields

$$
\left|T_{r}(n)\right| \ll n(\log n)^{-(G+H)} \ll \frac{N}{|k|(\log N)^{G+H}} .
$$

- On the contrary, if there are no such $a$ and $q$ then by Lemma 5.2 there must exist $a$ and $q$ with $(a, q)=1$ and $|q|^{2} \leq N^{s}(\log N)^{-H^{\prime}}$. Thus by (5.7) we get

$$
1 \leq|q|^{2} \leq(\log N)^{-H^{\prime}} \text { and } \quad\left|k^{s} \alpha_{s}-\frac{a}{q}\right| \leq \frac{(\log N)^{-H^{\prime} / 2}}{|q| N^{s / 2}} .
$$

Then, however,

$$
\left|k^{s} q\right|^{2} \leq(\log N)^{H^{\prime}+s K} \leq(\log N)^{K_{s}^{*}},
$$

and thus

$$
\left|\alpha_{s}-\frac{a}{k^{s} q}\right| \leq \frac{(\log N)^{H_{s}^{*}}}{|k|^{s}|q| N^{s}},
$$

which contradicts the assumption on $\alpha_{s}$.

Thus we have shown (5.5). Together with (5.2) and (5.4) we get

$$
\begin{aligned}
\sum_{|z|^{2} \leq N} e(\operatorname{tr}(f(z))) & \ll \sum_{r \in R}\left[\left|T_{r}(M)\right|+|k| \frac{(\log N)^{H}}{N} \sum_{n=1}^{M}\left|T_{r}(n)\right|\right]+\sqrt{N}|k| \\
& \ll \sum_{r \in R}\left[\frac{N}{|k|(\log N)^{G+H}}+\frac{1}{(\log N)^{G}} M\right]+\sqrt{N}|k| \\
& \ll \frac{N}{(\log N)^{G}},
\end{aligned}
$$

and the proposition is proven.

Now we have enough tools to proceed to the proof of the main theorem. 
6. Proof of Theorem 2.2. The proof of Theorem 2.2 will occupy the rest of the paper. We split it into several parts.

(1) We start in Section 6.1 by defining several useful parameters, and we show some connections between them.

(2) Then in Section 6.2 we rewrite the problem into one of estimating an exponential sum. This sum is finally transfered into one of type as in Proposition 5.1 or Lemma 5.3.

(3) We consider these sums according to the $b$-adic length of their arguments. Those of middle length, considered in Section 6.3, pose no problem. By "middle" we mean that there exists an upper and a lower bound for the $b$-adic length of the expansion. For those arguments with a long or short expansion we have to use different methods in Sections 6.4 and 6.5, respectively.

(4) Finally, we put everything together and get the result.

Throughout the proof we fix $N$ and the block $d_{1} \ldots d_{l}$. Furthermore, we set

$$
a:=\sum_{i=1}^{l} d_{i} b^{l-i}
$$

for abbreviation.

6.1. Defining parameters and explaining relations between them. Let $m$ be the unique positive integer such that

$$
\sum_{n \leq m-1} \ell\left(f\left(z_{n}\right)\right)<N \leq \sum_{n \leq m} \ell\left(f\left(z_{n}\right)\right),
$$

where $z_{n}:=\tau^{-1}(n-1)$ for $n \geq 1$. Furthermore, we denote by $M$ the maximum norm and by $J$ the maximum length of the $(b, \mathcal{D})$-ary expansion of $\left\lfloor f\left(z_{n}\right)\right\rfloor$ for $1 \leq n \leq m$, i.e.,

$$
M:=\max _{n \leq m}\left|z_{n}\right|^{2}, \quad J:=\max _{n \leq m} \ell\left(f\left(z_{n}\right)\right) .
$$

These will be of central interest for us.

Now we will use Lemma 3.2 to connect $m$ and $M$. We get

$$
\begin{aligned}
\left.\left|\log _{|b|^{2}} \max _{n \leq m}\right| z_{n}\right|^{2}-\ell\left(\max _{n \leq m} z_{n}\right) \mid & =\left|\log _{|b|^{2}} M-\ell\left(z_{m}\right)\right| \\
& =\left|\log _{|b|^{2}} M-\left\lfloor\log _{|b|^{2}} m\right\rfloor\right| \leq c, \\
M & \ll \gg m,
\end{aligned}
$$

where $\ll \gg$ means both $\ll$ and $\gg$.

For the connection of $M$ and $J$ we note that $|f(z)| \ll \gg|z|^{d}$. Thus by Lemma 3.2 we get 


$$
\begin{aligned}
&\left.\left|\log _{|b|^{2}} \max _{n \leq m}\right| f\left(z_{n}\right)\right|^{2}-J \mid\left.\ll \gg\left|\log _{|b|^{2}} \max _{n \leq m}\right| z_{n}\right|^{2 d}-J \mid \\
&=\left|\log _{|b|^{2}} M^{d}-J\right|, \\
& M \ll \gg|b|^{2 J / d} \leq c .
\end{aligned}
$$

Finally, we get the following relation between $M$ and $N$ :

$$
N=m J+\mathcal{O}(m)=c_{0} M \log _{q} M+\mathcal{O}(M),
$$

where $c_{0}$ is a positive constant depending on $d$ and $b$.

Next we want to split the sum on the right of (6.2) into parts where $f\left(z_{n}\right)$ has the same $b$-ary length. So let $I_{l}, I_{l+1}, \ldots, I_{J} \subset\{1, \ldots, m\}$ be such that

$$
n \in I_{j}: \Leftrightarrow \ell\left(f\left(z_{n}\right)\right) \geq j
$$

To estimate the size of these subsets we define $M_{j}(j=l, l+1, \ldots, J)$ to be the least integer such that any $z \in \mathbb{C}$ of norm greater than or equal to $M_{j}$ has length at least $j$, i.e.,

$$
M_{j}:=\max _{\ell(z)<j}|z|^{2}=\max _{n<|b|^{2(j-1)}}\left|z_{n}\right|^{2} .
$$

By the same arguments as in (6.3) we get $M_{j} \ll \gg|b|^{2 j / d}$. Furthermore, we set

$$
X_{j}:=M-M_{j} .
$$

6.2. Rewriting the problem. With the help of the parameters defined above we can easily rewrite our problem. Let $\mathcal{N}\left(f\left(z_{n}\right)\right)$ be the number of occurrences of the block $d_{1} \ldots d_{l}$ in the $b$-ary expansion of the integer part of $\left\lfloor f\left(z_{n}\right)\right\rfloor$. Then

$$
\left|\mathcal{N}\left(\theta_{q}(f) ; d_{1} \ldots d_{l}, N\right)-\sum_{n \leq m} \mathcal{N}\left(f\left(z_{n}\right)\right)\right| \leq 2 l m .
$$

Thus it suffices to show that

$$
\sum_{n \leq m} \mathcal{N}\left(f\left(z_{n}\right)\right)=\frac{N}{|\mathcal{D}|^{l}}+\mathcal{O}\left(\frac{N}{\log N}\right) .
$$

In order to count the occurrences of $d_{1} \ldots d_{l}$ in $\left\lfloor f\left(z_{n}\right)\right\rfloor$ properly, we introduce the indicator function of $\mathcal{F}_{a}$ (where $a$ is as in (6.1) and $\mathcal{F}_{a}$ is defined in (4.1)),

$$
\mathcal{I}_{a}(z)=\mathcal{I}(z)= \begin{cases}1, & z \in \mathcal{F}_{a} \\ 0, & \text { otherwise }\end{cases}
$$

Writing $f\left(z_{n}\right)$ in $(b, \mathcal{D})$-ary expansion for a fixed $n \in\{1, \ldots, m\}$, i.e.,

$$
f\left(z_{n}\right)=a_{r} b^{r}+a_{r-1} b^{r-1}+\cdots+a_{1} b+a_{0}+a_{-1} b^{-1}+\cdots,
$$


with $a_{i} \in \mathcal{D}$ for $i=r, r-1, \ldots$, we see that

$$
\mathcal{I}\left(b^{-j-1} f\left(z_{n}\right)\right)=1 \Leftrightarrow d_{1} \ldots d_{l}=a_{j-1} \ldots a_{j-l} .
$$

As every $I_{j}(l \leq j \leq J)$ consists of exactly those $f\left(z_{n}\right)$ whose $(b, \mathcal{D})$-ary expansion has length at least $j$, we get

$$
\sum_{n \leq m} \mathcal{N}(f(z))=\sum_{l \leq j \leq J} \sum_{n \in I_{j}} \mathcal{I}\left(\frac{f\left(z_{n}\right)}{b^{j+1}}\right) .
$$

For every $j$ there may be elements $z \in \mathbb{Z}[i]$ with $|z|^{2}<M_{j}$ but $\ell(z) \geq j$. By Lemma 3.2, they are only finitely many. Now by Lemma 3.1 we get

$$
\sum_{n \in I_{j}} 1=\sum_{\left|z_{n}\right|<M_{j}} 1+\sum_{M_{j} \leq\left|z_{n}\right|^{2}<M} 1 \sim \sum_{M_{j} \leq\left|z_{n}\right|^{2}<M} 1 .
$$

Therefore we can assume that there are no $z$ with $\ell(z) \geq j$ and $|z|^{2}<M_{j}$.

In order to estimate $\mathcal{I}(z)$ we use our considerations of Section 4. Noting that $\mathcal{F}_{a}$ can be covered by a set $I_{k, a}$ and an axis-parallel tube $P_{k, a}$ (cf. (4.2)), we have to consider how often the sequence $\left\{b^{-j-1} f\left(z_{n}\right)\right\}_{n \in I_{j}}$ hits each of these sets. The first one, $I_{k, a}$, is characterized by the Urysohn function $f_{a}(x, y)$ (cf. (4.3)) and for the axis-parallel tube $P_{k, a}$ we define

$$
\mathcal{E}_{j}:=\#\left\{n \in I_{j}: \varphi\left(\frac{f\left(z_{n}\right)}{b^{j+1}}\right) \in P_{k, a}\right\} .
$$

Thus for every $j \in\{l, l+1, \ldots, J\}$ we get

$$
\sum_{n \in I_{j}} \mathcal{I}\left(\frac{f\left(z_{n}\right)}{b^{j+1}}\right)=\sum_{n \in I_{j}} f_{a}\left(\varphi\left(\frac{f\left(z_{n}\right)}{b^{j+1}}\right)\right)+\mathcal{O}\left(\mathcal{E}_{j}\right) .
$$

We consider both terms on the right hand side of (6.6) separately. Starting with $f_{a}$, by Lemma 4.2 we get

$$
f_{a}\left(\varphi\left(\frac{f\left(z_{n}\right)}{b^{j+1}}\right)\right)=|b|^{-2 \ell(a)}+\sum_{\mathbf{0} \neq \mathbf{v} \in \mathbb{Z}^{2}} C\left(v_{1}, v_{2}\right) e\left(\mathbf{v} \cdot \varphi\left(\frac{f\left(z_{n}\right)}{b^{j+1}}\right)\right),
$$

where $\mathbf{v}=\left(v_{1}, v_{2}\right)$ and $C(\cdot, \cdot)$ is defined as in (4.5). We split the sum into those $\mathbf{v}$ with $\|\mathbf{v}\|_{\infty} \leq \Delta^{-1}$ and the rest. For $\|\mathbf{v}\|_{\infty}>\Delta^{-1}$ we apply (4.6) and estimate the $e(\cdot)$ function trivially to get

$$
\begin{aligned}
& \sum_{n \in I_{j}} f_{a}\left(\varphi\left(\frac{f\left(z_{n}\right)}{b^{j+1}}\right)\right) \\
& \quad \ll \frac{X_{j}}{|b|^{2 l}}+X_{j} \mu^{k} \Delta^{2}+\mu^{k} \sum_{\mathbf{0}<\|\mathbf{v}\|_{\infty} \leq \Delta^{-1}} \frac{1}{r(\mathbf{v})} \sum_{n \in I_{j}} e\left(\mathbf{v} \cdot \varphi\left(\frac{f\left(z_{n}\right)}{b^{j+1}}\right)\right) .
\end{aligned}
$$

To estimate $\mathcal{E}_{j}$ we use the Erdős-Turán-Koksma inequality (Lemma 3.3). By Lemma 4.1 we can split the tube $P_{k, a}$ into a family $\mathbf{R}_{j}$ of $\mu^{k}$ rectangles. 
As the discrepancy is defined on a rectangle (cf. [5, p. 5]), by Lemma 3.3 we get

$$
\begin{aligned}
& \text { (6.8) } \quad \mathcal{E}_{j} \ll \sum_{R \in \mathbf{R}_{j}} X_{j} \lambda_{2}(R)+X_{j} D_{X_{j}}\left(\left\{x_{n}\right\}\right) \\
& \ll X_{j} \sum_{R \in \mathbf{R}_{j}}\left(\lambda_{2}(R)+\frac{2}{H+1}+\sum_{0<\|\mathbf{h}\|_{\infty} \leq H} \frac{1}{r(\mathbf{v})}\left|\frac{1}{X_{j}} \sum_{n \in I_{j}} e\left(\mathbf{v} \cdot \varphi\left(\frac{f\left(z_{n}\right)}{b^{j+1}}\right)\right)\right|\right) .
\end{aligned}
$$

By the property (3) of $P_{k, a}$ described in Lemma 4.1 and possible overlappings of the rectangles in $\mathbf{R}_{j}$ we get

$$
\sum_{R \in \mathbf{R}_{j}} \lambda_{2}(R) \ll\left(\frac{\mu}{|b|^{2}}\right)^{k} .
$$

Thus (6.8) simplifies to

$$
\begin{aligned}
\mathcal{E}_{j} \ll & X_{j}\left(\left(\frac{\mu}{|b|^{2}}\right)^{k}+\frac{\mu^{k}}{H+1}\right. \\
& \left.+\frac{\mu^{k}}{X_{j}} \sum_{0<\|\mathbf{v}\|_{\infty} \leq H} \frac{1}{r(\mathbf{v})} \sum_{n \in I_{j}} e\left(\mathbf{v} \cdot \varphi\left(\frac{f\left(z_{n}\right)}{b^{j+1}}\right)\right)\right) .
\end{aligned}
$$

As both exponential sums in (6.7) and (6.8) are of the same shape, we define for short

$$
S(\mathbf{v}, j):=\sum_{n \in I_{j}} e\left(\mathbf{v} \cdot \varphi\left(\frac{f\left(z_{n}\right)}{b^{j+1}}\right)\right) .
$$

Plugging (6.7), (6.9), and (6.10) in (6.6) and subtracting the main part we get

$$
\begin{aligned}
\left|\sum_{n \in I_{j}} \mathcal{I}\left(\frac{f\left(z_{n}\right)}{b^{j+1}}\right)-\frac{X_{j}}{|b|^{2 l}}\right| \ll X_{j}\left(\mu^{k} \Delta^{2}+\frac{2 \mu^{k}}{H+1}+\left(\frac{\mu}{|b|^{2}}\right)^{k}\right) \\
+\sum_{\mathbf{0}<\|\mathbf{v}\|_{\infty} \leq \Delta^{-1}} \frac{\mu^{k}}{r(\mathbf{v})} S(\mathbf{v}, j)+\sum_{0<\|\mathbf{v}\|_{\infty} \leq H} \frac{\mu^{k}}{r(\mathbf{v})} S(\mathbf{v}, j) .
\end{aligned}
$$

In order to transfer the exponential sum from $\mathbb{Z}^{2}$ to $\mathbb{Z}[i]$ we use the same idea as Gittenberger and Thuswaldner in [6, p. 335]. Thus let

$$
\tau(z):=(\operatorname{tr}(z), \operatorname{tr}(b z))^{t}=\Xi \varphi(z),
$$

where $\Xi=V V^{t}$ and $V$ is the Vandermonde matrix

$$
V=\left(\begin{array}{ll}
1 & 1 \\
b & \bar{b}
\end{array}\right)
$$


Then

$$
\mathbf{v} \cdot \varphi\left(\frac{f(z)}{b^{j+1}}\right)=\mathbf{v} \Xi^{-1} \tau\left(\frac{f(z)}{b^{j+1}}\right)=\operatorname{tr}\left(\left(\widetilde{v}_{1}+b \widetilde{v}_{2}\right) \frac{f(z)}{b^{j+1}}\right),
$$

where $\left(\widetilde{v}_{1}, \widetilde{v}_{2}\right):=\mathbf{v} \Xi^{-1}$. Thus (6.10) transfers to

$$
\begin{aligned}
S(\mathbf{v}, j) & =\sum_{n \in I_{j}} e\left(\operatorname{tr}\left(\left(\widetilde{v}_{1}+b \widetilde{v}_{2}\right) \frac{f\left(z_{n}\right)}{b^{j+1}}\right)\right) \\
& \ll \sum_{M_{j} \leq|z|^{2}<M_{j}+X_{j}} e\left(\operatorname{tr}\left(\left(\widetilde{v}_{1}+b \widetilde{v}_{2}\right) \frac{f(z)}{b^{j+1}}\right)\right),
\end{aligned}
$$

where we have used the fact that $\left|I_{j}\right| \ll \gg X_{j}$ together with the definition of $X_{j}$ in (6.4).

We assume that $k$ and $H$ are such that $\Delta^{-1}, H \ll \log N$, which is possible since $\Delta$ depends on $k$ (cf. (4.4)). The values of $k$ and $H$ are chosen later depending on $j$.

In the following subsections we want to consider the different sums $S(\mathbf{v}, j)$ according to the size of $j$. We therefore consider separately the three intervals

$$
\begin{aligned}
l & \leq j \leq l+C_{l} \log \log N, \\
l+C_{l} \log \log N & <j \leq J-C_{u} \log \log N, \\
J-C_{u} \log \log N & <j \leq J,
\end{aligned}
$$

where $C_{l}$ and $C_{u}$ are sufficiently large constants.

6.3. A first estimation of $S(\mathbf{v}, j)$. We start with the $j$ satisfying (6.14). Assume first that there are two Gaussian integers $a$ and $q$ such that

$$
\begin{aligned}
& \left|\frac{\widetilde{v}_{1}+b \widetilde{v}_{2}}{b^{j}} \alpha_{d}-\frac{a}{q}\right| \leq \frac{1}{|q|^{2}}, \\
& \left(\log X_{j}\right)^{H} \leq|q|^{2} \leq X_{j}^{d}\left(\log X_{j}\right)^{-H},
\end{aligned}
$$

with $G=3$ and $H=2^{d+2} G+2^{3(d+2)}$. Then we apply Lemma 5.3 to get

$$
S(\mathbf{v}, j) \ll X_{j}\left(\log X_{j}\right)^{-G} .
$$

Now we show that (6.16) holds for all $j$ satisfying (6.14). Indeed, otherwise by Lemma 5.2 there are $a, q \in \mathbb{Z}[i]$ such that

$$
\begin{aligned}
& (a, q)=1, \quad 1 \leq|q|^{2} \leq X_{j}^{d}\left(\log X_{j}\right)^{-H}, \\
& \left|\frac{\widetilde{v}_{1}+b \widetilde{v}_{2}}{b^{j}} \alpha_{d}-\frac{a}{q}\right| \leq \frac{\left(\log X_{j}\right)^{H}}{|q| X_{j}^{d / 2}} \leq \frac{1}{|q|^{2}} .
\end{aligned}
$$

We distinguish two cases depending on the size of $|q|^{2}$. Assume first that $2 \leq|q|^{2} \leq\left(\log X_{j}\right)^{H}$. Then 


$$
\left|\frac{\widetilde{v}_{1}+b \widetilde{v}_{2}}{b^{j}} \alpha_{d}\right|>\frac{1}{|q|}-\frac{1}{|q|^{2}} \geq \frac{1}{2|q|} \gg\left(\log X_{j}\right)^{-H}
$$

and therefore

$$
|b|^{j} \ll\left|\left(\widetilde{v}_{1}+b \widetilde{v}_{2}\right) \alpha_{d}\right|\left(\log X_{j}\right)^{H} \ll(\log N)\left(\log X_{j}\right)^{H},
$$

which contradicts (6.14) for $C_{l}$ sufficiently large.

We denote by $\|z\|$ the distance of the norm of $z$ over $\mathbb{Q}$ to the nearest integer, i.e.,

$$
\|z\|:=\left.\min _{n \in \mathbb{Z}}|| z\right|^{2}-n \mid .
$$

Now if $|q|^{2}=1$ then $q=1$ and $\left\|\left(\widetilde{v}_{1}+b \widetilde{v}_{2}\right)\left(b^{-j}\right) \alpha_{d}\right\|<X_{j}^{d}\left(\log X_{j}\right)^{-2 H}$. If $\left|\left(\widetilde{v}_{1}+b \widetilde{v}_{2}\right)\left(b^{-j}\right) \alpha_{d}\right|^{2}>\sqrt{2} / 2$ then

$$
|b|^{2 j} \ll\left|\left(\widetilde{v}_{1}+b \widetilde{v}_{2}\right) \alpha_{d}\right| \ll \log N,
$$

which contradicts (6.14) for $C_{l}$ sufficiently large.

On the other hand, if $\left|\left(\widetilde{v}_{1}+b \widetilde{v}_{2}\right) b^{-j} \alpha_{d}\right|<\sqrt{2} / 2$ we get

$$
\left|\left(\widetilde{v}_{1}+b \widetilde{v}_{2}\right) b^{-j} \alpha_{d}\right|^{2}=\left\|\left(\widetilde{v}_{1}+b \widetilde{v}_{2}\right) b^{-j} \alpha_{d}\right\|<X_{j}^{d}\left(\log X_{j}\right)^{-2 H},
$$

which implies that

$$
|b|^{2 j} \gg\left|\left(\widetilde{v}_{1}+b \widetilde{v}_{2}\right) \alpha_{d}\right|^{2} X_{j}^{d}\left(\log X_{j}\right)^{-2 H},
$$

contradicting our assumption on $C_{u}$ in (6.14).

Thus for $j$ such that (6.14) holds we get

$$
S(\mathbf{v}, j) \ll X_{j}\left(\log X_{j}\right)^{-G} .
$$

Plugging this into (6.11) yields

$$
\begin{aligned}
\left|\sum_{n \in I_{j}} \mathcal{I}\left(\frac{f\left(z_{n}\right)}{b^{j+1}}\right)-\frac{X_{j}}{|b|^{2 l}}\right| \ll X_{j}\left(\mu^{k} \Delta^{2}+\frac{\mu^{k}}{V+1}+\left(\frac{\mu}{|b|^{2}}\right)^{k}\right. & \\
+ & \left.\frac{\mu^{k}}{\left(\log X_{j}\right)^{3}}\left\{\sum_{\mathbf{0}<\|\mathbf{v}\|_{\infty} \leq \Delta^{-1}}+\sum_{0<\|\mathbf{v}\|_{\infty} \leq V}\right\} \frac{1}{r(\mathbf{v})}\right) .
\end{aligned}
$$

Now we can choose $k$ and $H$ so that $\Delta^{-1}, H \ll \log N$ as claimed in our assumption above. For $j$ as in (6.14) together with the definition of $\Delta$ in (4.4) we set

$$
k:=C_{k} \log \log X_{j}, \quad H:=\mu^{k} \log X_{j}, \quad \Delta^{-1}=\frac{\left(\log X_{j}\right)^{C_{k} \log |b|}}{2 c_{\Delta}},
$$

for $C_{k}$ an arbitrary constant. Furthermore we define $C_{\mu}>1$ to be such that

$$
C_{\mu} \mu=|b|^{2} \text {. }
$$


Then for $j$ as in (6.14) we get

$$
\begin{aligned}
\mid \sum_{n \in I_{j}} \mathcal{I}\left(\frac{f\left(z_{n}\right)}{b^{j+1}}\right) & -\frac{X_{j}}{|b|^{2 l}} \mid \\
& \ll X_{j}\left(\left(\log X_{j}\right)^{-1}+\left(\log X_{j}\right)^{-2}\left(\log \log X_{j}\right)^{2}\right) \ll \frac{X_{j}}{j} .
\end{aligned}
$$

We will prove the same estimate for smaller and larger $j$.

6.4. Estimating the exponential sum for long b-ary expansions. We now concentrate on values of $j$ satisfying (6.15).

In this case we start with the same assumptions for $\Delta^{-1}$ and $H$ as above, i.e., $\Delta^{-1}, H \ll \log N$. For every $j$ such that (6.16) holds, by Lemma 5.3 we get

$$
S(\mathbf{v}, j) \ll X_{j}\left(\log X_{j}\right)^{-G} .
$$

If (6.16) does not hold, then for every $j$ in (6.15) with $|b|^{j / d} \ll X_{j} \ll|b|^{J / d}$,

$$
\begin{aligned}
0 & \ll\left|\widetilde{v}_{1}+b \widetilde{v}_{2}\right||b|^{-j / 2 d} \ll\left|f^{\prime}(z)\right| \ll\left|\widetilde{v}_{1}+b \widetilde{v}_{2}\right||b|^{J-j-j / 2 d} \\
& \ll\left|\widetilde{v}_{1}+b \widetilde{v}_{2}\right||b|^{-j / 2 d}(\log N)^{\widetilde{C}_{2}} .
\end{aligned}
$$

We use the inequalities (6.21) to apply Lemma 3.6 with

$$
F=\operatorname{tr}\left(\left(\widetilde{v}_{1}+b \widetilde{v}_{2}\right) \frac{f\left(z_{n}\right)}{b^{j+1}}\right)
$$

$m=\left|\widetilde{v}_{1}+b \widetilde{v}_{2}\right||b|^{-j / d}$, and $\delta=\left|\widetilde{v}_{1}+b \widetilde{v}_{2}\right||b|^{-j / d}(\log N)^{\widetilde{C}_{2}}$. Thus for $j$ as in (6.15) and for $\sigma=2 G$ we get

$$
\begin{aligned}
S(\mathbf{v}, j) & \ll \frac{\sqrt{X_{j}}}{\left|\widetilde{v}_{1}+b \widetilde{v}_{2}\right||b|^{-j / d}}+\frac{X_{j}}{\left(\log X_{j}\right)^{\sigma / 2}}+s \frac{3-\delta}{1-\delta} \sqrt{X_{j}\left(\log X_{j}\right)^{\sigma}} \\
& \ll \frac{\sqrt{X_{j}}|b|^{j / d}}{\left|\widetilde{v}_{1}+b \widetilde{v}_{2}\right|}+\frac{X_{j}}{\left(\log X_{j}\right)^{G}} .
\end{aligned}
$$

Plugging this into (6.11) yields

$$
\begin{aligned}
& \left|\sum_{n \in I_{j}} \mathcal{I}\left(\frac{f\left(z_{n}\right)}{b^{j+1}}\right)-\frac{X_{j}}{|b|^{2 l}}\right| \ll X_{j}\left(\mu^{k} \Delta^{2}+\frac{2 \mu^{k}}{H+1}+\left(\frac{\mu}{|b|^{2}}\right)^{k}\right. \\
& \left.\quad+\frac{\mu^{k}}{X_{j}}\left\{\sum_{\mathbf{0}<\|\mathbf{v}\|_{\infty} \leq \Delta^{-1}}+\sum_{\mathbf{0}<\|\mathbf{v}\|_{\infty} \leq H}\right\} \frac{1}{r(\mathbf{v})}\left(\frac{\sqrt{X_{j}}|b|^{j / d}}{\left|\widetilde{v}_{1}+b \widetilde{v}_{2}\right|}+\frac{X_{j}}{\left(\log X_{j}\right)^{3}}\right)\right) .
\end{aligned}
$$

Now we set

$k:=\max \left(1, \frac{\frac{1}{2} \log X_{j}+\log 4 C_{\Delta}^{2}-\frac{j}{d} \log |b|}{\log C_{\mu}}\right), \quad H:=\mu^{k} \log X_{j}, \quad \Delta^{-1}=\frac{|b|^{k}}{2 c_{\Delta}}$. 
This yields

$$
\mu^{k} \Delta^{2}=\frac{|b|^{j / d}}{\sqrt{X_{j}}}, \quad \mu^{k} \leq|b|^{2 k} \ll\left(\frac{X_{j}}{|b|^{2 j / d}}\right)^{\frac{\log |b|}{\log C_{\mu}}}, \quad\left(\frac{\mu}{|b|^{2}}\right)^{k}=\frac{1}{C_{\mu}^{k}} \ll \frac{|b|^{j / d}}{\sqrt{X_{j}}} .
$$

Furthermore,

$$
\left|\widetilde{v}_{1}+b \widetilde{v}_{2}\right|=\left|(1, b)\left(v_{1}, v_{2}\right)^{t} \Xi^{-1}\right| \gg\left|\left(v_{1}, v_{2}\right)^{t}\right| \gg \sqrt{v_{1} v_{2}} .
$$

Putting all this in (6.23) yields

$$
\begin{aligned}
& \left|\sum_{n \in I_{j}} \mathcal{I}\left(\frac{f\left(z_{n}\right)}{b^{j+1}}\right)-\frac{X_{j}}{|b|^{2 l}}\right| \\
& \ll \sqrt{X_{j}}|b|^{j / d}+\frac{X_{j}}{j}+\left(\frac{X_{j}}{|b|^{2 j / d}}\right)^{\frac{\log |b|}{\log C_{\mu}}}\left(\sqrt{X_{j}}|b|^{j / d}+X_{j}\left(\log X_{j}\right)^{-3}\right)
\end{aligned}
$$

for $j$ as in (6.15).

6.5. Iterative estimation for short b-ary expansions. We finally consider the case of $j$ satisfying (6.13). This will be the hardest part, as by our assumptions on $H$ and $\Delta^{-1}$ we have

$$
\left|\widetilde{v}_{1}+b \widetilde{v}_{2}\right| \ll \gg|b|^{j} .
$$

We adopt the idea of Nakai and Shiokawa [22, p. 278ff] applying Proposition 5.1 iteratively. If there is no such $s$ as assumed in that proposition, we will apply Lemmas 3.6 and 3.7.

By the assumption $j \leq l+C_{l} \log \log N$ we get

$$
|b|^{j} \leq(\log N)^{C_{l} \log |b|+o(1)} .
$$

We define $g$ to be the polynomial

$$
g(z):=\frac{\widetilde{v}_{1}+b \widetilde{v}_{2}}{b^{j}} f(z)
$$

with coefficients

$$
\beta_{i}=\frac{\widetilde{v}_{1}+b \widetilde{v}_{2}}{b^{j}} \alpha_{i}, \quad i=0,1, \ldots, d .
$$

Now we start the application of Proposition 5.1. We assume first that $1 \leq s \leq d$. Then we set

$$
H_{d}=H_{d}^{*}+C_{1} \log |b|+1, \quad H_{d}^{*}=2^{3(d+2)}+2^{d+3} G
$$

and define $H_{r}^{*}, H_{r}$, and $h_{r}(1 \leq r<d)$ inductively by

$$
\begin{aligned}
H_{r}^{*} & =2^{3(r+2)}+2^{r+3}\left(G+H_{r+1}\right)+2 r \sum_{i=r+1}^{d} H_{r}, \\
H_{r} & =H_{r}^{*}+2\left(C_{1} \log |b|+1\right), \\
h_{r} & =H_{r}^{*}+C_{1} \log |b|+1 .
\end{aligned}
$$


Let $j$ be such that $l \leq j \leq l+C_{l} \log \log N$ and there are coprime pairs $\left(a_{d}, q_{d}\right), \ldots,\left(a_{s+1}, q_{s+1}\right)$ of Gaussian integers such that

$$
1 \leq\left|q_{r}\right|^{2} \leq\left(\log X_{j}\right)^{2 h_{r}} \quad \text { and } \quad\left|\alpha_{r}-\frac{a_{r}}{q_{r}}\right| \leq \frac{\left(\log X_{j}\right)^{h_{r}}}{\left|q_{r}\right| X_{j}^{r / 2}} \quad(s<r \leq d),
$$

but there is no pair $\left(a_{s}, q_{s}\right)$ such that

$$
1 \leq\left|q_{s}\right|^{2} \leq\left(\log X_{j}\right)^{2 h_{s}} \quad \text { and } \quad\left|\alpha_{s}-\frac{a_{s}}{q_{s}}\right| \leq \frac{\left(\log X_{j}\right)^{h_{s}}}{\left|q_{s}\right| X_{j}^{s / 2}}
$$

We denote the set of all such $j$ by $\mathbb{J}_{s}$.

For every $j \in \mathbb{J}_{s}$ we have

$$
1 \leq\left|b^{j} q_{r}\right| \leq\left(\log X_{j}\right)^{2 H_{r}} \quad \text { and } \quad\left|\beta_{r}-\frac{\left(\widetilde{v}_{1}+b \widetilde{v}_{2}\right) a_{r}}{b^{j} q_{r}}\right| \leq \frac{\left(\log X_{j}\right)^{H_{r}}}{\left|b^{j} q_{r}\right| X_{j}^{r / 2}}
$$

for $s<r \leq d$, but there is no pair $\left(A_{s}, Q_{s}\right)$ of coprime Gaussian integers such that

$$
1 \leq\left|Q_{s}\right| \leq\left(\log X_{j}\right)^{2 H_{s}^{*}} \quad \text { and } \quad\left|\beta_{r}-\frac{A_{s}}{Q_{s}}\right| \leq \frac{\left(\log X_{j}\right)^{H_{s}^{*}}}{\left|Q_{s}\right| X_{j}^{s / 2}}
$$

since, if there were such $A_{s}$ and $Q_{s}$, we would get

$$
1 \leq\left|\left(\widetilde{v}_{1}+b \widetilde{v}_{2}\right) Q_{s}\right|^{2} \leq\left(\log X_{j}\right)^{2 H_{s}^{*}+t} \leq\left(\log X_{j}\right)^{2 h_{s}},
$$

and together with (6.25),

$$
\left|\alpha_{s}-\frac{b^{j} A_{s}}{\widetilde{v}_{1}+b \widetilde{v}_{2} Q_{s}}\right| \leq \frac{\left(\log X_{j}\right)^{H_{s}^{*}+C_{1} \log |b|+1}}{\left|\left(\widetilde{v}_{1}+b \widetilde{v}_{2}\right) Q_{s}\right| X_{j}^{s / 2}} \leq \frac{\left(\log X_{j}\right)^{h_{s}}}{\left|\left(\widetilde{v}_{1}+b \widetilde{v}_{2}\right) Q_{s}\right| X_{j}^{s / 2}},
$$

which contradicts the assumption that $j \in \mathbb{J}_{s}$.

Thus an application of Proposition 5.1 with $H_{i}, H_{i}^{*}$ and $K_{i}=2 H_{i}$, $K_{i}^{*}=2 H_{i}^{*}$ yields

$$
S(\mathbf{v}, j) \ll X_{j}\left(\log X_{j}\right)^{-G}
$$

for all $j \in \mathbb{J}_{1} \cup \cdots \cup \mathbb{J}_{d}$.

Now we denote by $\mathbb{J}_{0}$ all positive integers $j$ with $l \leq j \leq l+C_{1} \log \log N$ and $j \notin \mathbb{J}_{1} \cup \cdots \cup \mathbb{J}_{d}$. It remains to estimate $S(\mathbf{v}, j)$ for such $j$. To this end, we will apply Lemmas 3.6 and 3.7.

For $j \in \mathbb{J}_{0}$ there exist coprime pairs $\left(a_{r}, q_{r}\right)$ of Gaussian integers such that

$$
1 \leq\left|q_{r}\right|^{2} \leq\left(\log X_{j}\right)^{2 h_{r}} \quad \text { and } \quad\left|\alpha_{r}-\frac{a_{r}}{q_{r}}\right| \leq \frac{\left(\log X_{j}\right)^{h_{r}}}{\left|q_{r}\right| X_{j}^{r / 2}} \quad(1 \leq r \leq d) .
$$

We set $\Omega_{r}=\alpha_{r}-a_{r} / q_{r}$ for $r=1, \ldots, d$, and $a=\operatorname{gcd}\left(a_{1}, \ldots, a_{d}\right)$ and 
$q=\operatorname{lcm}\left(q_{1}, \ldots, q_{d}\right)$. Furthermore, we define $c_{r}$ by

$$
\frac{a_{r}}{q_{r}}=\frac{a}{q} c_{r} \quad(r=1, \ldots, d) .
$$

Then we can rewrite the exponential sum as follows:

$$
\begin{aligned}
S(\mathbf{v}, j)= & \sum_{n \in I_{j}} e\left(\operatorname{tr}\left(\left(\widetilde{v}_{1}+b \widetilde{v}_{2}\right) \frac{f\left(z_{n}\right)}{b^{j+1}}\right)\right) \\
= & \sum_{\lambda \in r\left(b^{j+1} q\right)} e\left(\operatorname{tr}\left(\frac{\widehat{v} a}{b^{j+1} q} \sum_{k=1}^{d} c_{k} \lambda^{k}\right)\right) \\
& \times \sum_{\substack{\mu \\
\exists n \in I_{j}: \mu q+\lambda=z_{n}}} e\left(\operatorname{tr}\left(\frac{\widehat{v}}{b^{j+1}} \sum_{k=1}^{d} \Omega_{k}(\mu q+\lambda)^{k}\right)\right),
\end{aligned}
$$

where $r\left(b^{j+1} q\right)$ denotes a complete system of residues modulo $b^{j+1} q$ and $\widehat{v}:=\widetilde{v}_{1}+b \widetilde{v}_{2}$.

We first consider the second sum. Let $R_{0}=\left(b^{j+1} q\right) \cdot\{\alpha+\beta i: 0 \leq \alpha, \beta$ $\leq 1\}$ and let $T_{0}$ be the set of translation vectors such that $R_{0}$ tiles $\mathbb{Z}^{2}$, i.e., $T_{0}:=\left\{\left(b^{j+1} q\right) z: z \in \mathbb{Z}[i]\right\}$. Furthermore we set

$$
T:=\left\{t \in T_{0}:\left(R_{0}+t\right) \cap\left\{z_{n}: n \in I_{j}\right\} \neq \emptyset\right\} .
$$

Then it is clear that $|T| \ll X_{j}\left|b^{j+1} q\right|^{-2}$. Furthermore, let $\mathcal{T}$ denote the area covered by the translates corresponding to $T$, i.e.,

$$
\mathcal{T}:=\bigcup_{t \in T}\left(R_{0}+t\right)
$$

For a fixed $\lambda \in R_{0} \cap \mathbb{Z}[i]$ we get

$$
\begin{aligned}
& \sum_{\substack{\mu \\
\exists n \in I_{j}: \mu q+\lambda=z_{n}}} e\left(\operatorname{tr}\left(\frac{\widehat{v}}{b^{j+1}} \sum_{k=1}^{d} \Omega_{k}(\mu q+\lambda)^{k}\right)\right) \\
& \leq \sum_{\mu \in T} e\left(\operatorname{tr}\left(\frac{\widehat{v}}{b^{j+1}} \sum_{k=1}^{d} \Omega_{k}(\mu q+\lambda)^{k}\right)\right) .
\end{aligned}
$$

Now we want to apply Lemma 3.5 together with the idea in the proof of Lemma 3.6. Therefore we set

$$
F_{\lambda}(x, y):=e\left(\operatorname{tr}\left(\frac{\widehat{v}}{b^{j+1}} \sum_{k=1}^{d} \Omega_{k}((x+i y) q+\lambda)^{k}\right)\right) .
$$


Then

$$
\begin{aligned}
\frac{\partial F_{\lambda}(x, y)}{\partial x} \ll \gg \frac{\partial F_{\lambda}(x, y)}{\partial y} & \ll \frac{\widehat{v}}{|b|^{j}} \sum_{k=1}^{d} k|q| \frac{\left(\log X_{j}\right)^{H_{k}}}{q_{k} X_{j}^{k / 2}} X_{j}^{(k-1) / 2} \\
& \ll \frac{\widehat{v}}{|b|^{j}} X_{j}^{-1 / 2}|q|\left(\log X_{j}\right)^{H_{1}^{*}} .
\end{aligned}
$$

As in the proof of Lemma 3.6 we first consider a single square. Set $D_{\nu}:=\{z=x+i y \in \mathbb{Z}[i]:-\nu \leq x, y \leq \nu\}$. An application of Lemma 3.5 yields

$$
\sum_{x+i y \in D_{\nu}} F_{\lambda}(x, y)=\sum_{x=-\nu}^{\nu} \sum_{y=-\nu}^{\nu} F_{\lambda}(x, y)=\int_{-\nu}^{\nu} \int_{-\nu}^{\nu} F_{\lambda}(x, y) d x d y+\mathcal{O}(\nu) .
$$

Now we again want to split $\mathcal{T}$ into squares. Note that we have assumed that $\left|I_{j}\right|=X_{j}$ and thus we can consider $I_{j}$ as an annulus $\left\{z \in \mathbb{C}: M_{j} \leq\right.$ $\left.|z|^{2}<M\right\}$. Thus we choose a $\sigma>0$ and tessellate $\mathcal{T}$ by squares of side length $\sqrt{|T| /(\log |T|)^{\sigma}}$. Then we can glue all squares in the interior of $\mathcal{T}$ together and estimate their contribution on the boundary to the error term. Thus we get

$$
\sum_{x+i y \in T} F_{\lambda}(x, y)=\iint_{\mathcal{T}} F_{\lambda}(x, y) d x d y+\mathcal{O}\left(\frac{|T|}{(\log |T|)^{\sigma / 2}}\right) .
$$

Putting everything together yields

$$
\begin{aligned}
& S(\mathbf{v}, j)=\sum_{n \in I_{j}} e\left(\operatorname{tr}\left(\left(\widetilde{v}_{1}+b \widetilde{v}_{2}\right) \frac{f\left(z_{n}\right)}{b^{j+1}}\right)\right) \\
& =\sum_{\lambda \in r\left(b^{j} q\right)} e\left(\operatorname{tr}\left(\frac{\nu a}{b^{j} q} \sum_{k=1}^{d} c_{k} \lambda^{k}\right)\right)\left\{\iint_{\mathcal{T}} F_{\lambda}(x, y) d x d y+\mathcal{O}\left(\frac{|T|}{(\log |T|)^{\sigma / 2}}\right)\right\} \\
& =\sum_{\lambda \in r\left(b^{j} q\right)} e\left(\operatorname{tr}\left(\frac{\nu a}{b^{j} q} \sum_{k=1}^{d} c_{k} \lambda^{k}\right)\right) \frac{1}{\left|b^{j+1} q\right|^{2}} \iint_{M_{j} \leq|z|^{2}<M} G(z) d z+\mathcal{O}\left(\frac{X_{j}}{\left(\log X_{j}\right)^{\sigma}}\right),
\end{aligned}
$$

where

$$
G(z):=e\left(\operatorname{tr}\left(\frac{\widehat{v}}{b^{j+1}} \sum_{k=1}^{d} \Omega_{k} z^{k}\right)\right) .
$$

Finally, we define rationals $R_{i} / Q \in \mathbb{Q}(i)$ for $i=1, \ldots, d$ by

$$
\frac{R_{i}}{Q}=\frac{\widehat{v}}{b^{j}} \frac{a c_{i}}{q} .
$$

Thus estimating the integral trivially and noting that

$$
N(\widehat{v} Q)=N\left(b^{j+1} R_{i} q_{i} / a_{i}\right) \ll \gg N\left(b^{j+1} R_{i} \alpha_{i}^{-1}\right) \ll \gg N\left(b^{j+1} R_{i}\right) \gg N\left(b^{j+1}\right)
$$


we get, by an application of Lemma 3.7,

$$
\begin{aligned}
S(\mathbf{v}, j) & =\sum_{n \in I_{j}} e\left(\operatorname{tr}\left(\left(\widetilde{v}_{1}+b \widetilde{v}_{2}\right) \frac{f\left(z_{n}\right)}{b^{j+1}}\right)\right) \\
& \ll \frac{\left|b^{j} q\right|^{2}}{N(Q)}(N(Q))^{1-1 / d+\varepsilon} \frac{X_{j}}{\left|b^{j} q\right|^{2}}+\frac{X_{j}}{\left(\log X_{j}\right)^{\sigma}} \\
& \ll X_{j}\left(\left(N\left(\widehat{v}^{-1} b^{j+1}\right)\right)^{-1 / d+\varepsilon}+\left(\log X_{j}\right)^{-\sigma}\right) .
\end{aligned}
$$

Plugging this into (6.11) yields

$$
\begin{aligned}
&\left|\sum_{n \in I_{j}} \mathcal{I}\left(\frac{f\left(z_{n}\right)}{b^{j+1}}\right)-\frac{X_{j}}{|b|^{2 l}}\right| \ll X_{j}\left(\mu^{k} \Delta^{2}+\frac{2 \mu^{k}}{H+1}+\left(\frac{\mu}{|b|^{2}}\right)^{k}\right. \\
& \quad+\mu^{k}\left\{\sum_{\mathbf{0}<\|\mathbf{v}\|_{\infty} \leq \Delta^{-1}}+\sum_{\mathbf{0}<\|\mathbf{v}\|_{\infty} \leq H}\right\} \frac{1}{r(\mathbf{v})}\left(\left(N\left(\widehat{v}^{-1} b^{j+1}\right)\right)^{-1 / d+\varepsilon}\right. \\
&\left.\left.\quad+\left(\log X_{j}\right)^{-\sigma}\right)\right) .
\end{aligned}
$$

Now we set $\sigma, k$, and $H$ to the same values as in (6.19) and get, together with (4.4),

$$
\sigma:=G, \quad k:=C_{k} \log \log X_{j}, \quad H:=\mu^{k} \log X_{j}, \quad \Delta^{-1}=\frac{\left(\log X_{j}\right)^{C_{k} \log |b|}}{2 c_{\Delta}},
$$

for $C_{k}$ an arbitrary constant.

We note that

$$
\left|\widetilde{v}_{1}+b \widetilde{v}_{2}\right|=\left|(1, b)\left(v_{1}, v_{2}\right)^{t} \Xi^{-1}\right| \ll\left|\left(v_{1}, v_{2}\right)\right| \ll r(\mathbf{v}) .
$$

At this point we have to distinguish two cases according to the size of $d$.

- $d=1$ : By noting that $\Delta^{-1}, H \ll \log N$ we get

$$
\begin{aligned}
\sum_{\mathbf{0}<\|\mathbf{v}\|_{\infty} \leq \log N} \frac{1}{r(\mathbf{v})}\left(N\left(\widehat{v}^{-1} b^{j+1}\right)\right)^{-1+\varepsilon} & \ll \sum_{\mathbf{0}<\|\mathbf{v}\|_{\infty} \leq \log N} \frac{\left|\widetilde{v}_{1}+b \widetilde{v}_{2}\right|}{|b|^{(2-\varepsilon)(j+1) / d}} \\
& \ll \frac{(\log N)^{4}}{|b|^{2 j / d}} .
\end{aligned}
$$

- $d \geq 2$ : In this case

$$
r(\mathbf{v})^{-1} \ll\left|\widetilde{v}_{1}+b \widetilde{v}_{2}\right|^{-1} \ll\left|\widetilde{v}_{1}+b \widetilde{v}_{2}\right|^{-2 / d} .
$$


This together with $\Delta^{-1}, H \ll \log N$ yields

$$
\begin{aligned}
\sum_{\mathbf{0}<\|\mathbf{v}\|_{\infty} \leq \log N} \frac{1}{r(\mathbf{v})}\left(N\left(\widehat{v}^{-1} b^{j+1}\right)\right)^{-1 / d+\varepsilon} & \ll \sum_{\mathbf{0}<\|\mathbf{v}\|_{\infty} \leq \log N} \frac{1}{|b|^{(2-\varepsilon)(j+1) / d}} \\
& \ll \frac{(\log N)^{2}}{|b|^{2 j / d}} .
\end{aligned}
$$

Therefore in any case

$$
\sum_{\mathbf{0}<\|\mathbf{v}\|_{\infty} \leq \log N} \frac{1}{r(\mathbf{v})}\left(N\left(\widehat{v}^{-1} b^{j+1}\right)\right)^{-1 / d+\varepsilon} \ll \frac{(\log N)^{4}}{|b|^{2 j / d}} .
$$

Putting this all in (6.29) yields

$$
\begin{aligned}
\mid \sum_{n \in I_{j}} \mathcal{I} & \left(\frac{f\left(z_{n}\right)}{b^{j+1}}\right)-\frac{X_{j}}{|b|^{2 l}} \mid \\
& \ll X_{j}\left(\left(\log X_{j}\right)^{-1}+\frac{(\log N)^{4} \log X_{j}}{|b|^{2 j / d}}\right) \ll \frac{X_{j}}{j}+X_{j} \frac{(\log N)^{5}}{|b|^{2 j / d}} .
\end{aligned}
$$

6.6. Putting all together. Now we have reached the final state of the proof. In order to finish we will put (6.20), (6.24), and (6.30) together and consider the corresponding intervals, which are described in (6.14), (6.15), and (6.13), respectively. Thus

$$
\sum_{l \leq j \leq J}\left|\sum_{n \in I_{j}} \mathcal{I}\left(\frac{f\left(z_{n}\right)}{b^{j+1}}\right)-\frac{X_{j}}{|b|^{2 l}}\right| \ll S_{1}+S_{2}+S_{3},
$$

where

$$
\begin{aligned}
S_{1}= & \sum_{l \leq j \leq J} \frac{X_{j}}{j}, \\
S_{2}= & \sum_{l \leq j \leq l+C_{l} \log \log N} X_{j} \frac{(\log N)^{5}}{|b|^{2 j / d}}, \\
S_{3}= & \sum_{J-C_{u} \log \log N \leq j \leq J} \sqrt{X_{j}}|b|^{j / d} \\
& +\left(\frac{X_{j}}{|b|^{2 j / d}}\right)^{\frac{\log |b|}{\log C_{\mu}}}\left(\sqrt{X_{j}}|b|^{j / d}+X_{j}\left(\log X_{j}\right)^{-3}\right) .
\end{aligned}
$$

We easily get

$$
S_{1} \ll M \text {. }
$$

The second sum is a bit more delicate and simplifies to

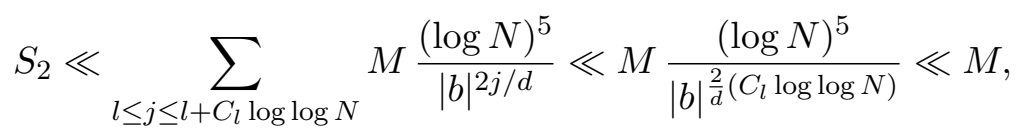


where we have assumed that $C_{l} \geq 5$. For the third sum we have to work a little more to get

$$
\begin{aligned}
S_{3} & \ll \sum_{J-C_{u} \log \log N \leq j \leq J} \sqrt{M}|b|^{j / d}+\left(\frac{M}{|b|^{2 j / d}}\right)^{\frac{\log |b|}{\log C_{\mu}}}\left(\sqrt{M}|b|^{j / d}+M\right) \\
& \ll \sqrt{M}|b|^{J / d}+\left(\frac{M}{|b|^{2 J / d}}\right)^{\frac{\log |b|}{\log C_{\mu}}}\left(\sqrt{M}|b|^{J / d}+M\right) \ll M .
\end{aligned}
$$

Putting this in (6.31) yields

$$
\sum_{l \leq j \leq J}\left|\sum_{n \in I_{j}} \mathcal{I}\left(\frac{f\left(z_{n}\right)}{b^{j+1}}\right)-\frac{X_{j}}{|b|^{2 l}}\right| \ll M \ll \frac{N}{\log N}
$$

and the main theorem is proven.

\section{References}

[1] T. M. Apostol, Mathematical Analysis: a Modern Approach to Advanced Calculus, Addison-Wesley, Reading, MA, 1957.

[2] G. I. Arkhipov, V. N. Chubarikov, and A. A. Karatsuba, Trigonometric Sums in Number Theory and Analysis, de Gruyter Exp. Math. 39, de Gruyter, Berlin, 2004.

[3] H. Davenport and P. Erdős, Note on normal decimals, Canad. J. Math. 4 (1952), $58-63$.

[4] M. M. Dodson and S. Kristensen, Hausdorff dimension and Diophantine approximation, in: Fractal Geometry and Applications: a jubilee of Benoit Mandelbrot. Part 1, Proc. Sympos. Pure Math. 72, Amer. Math. Soc., Providence, RI, 2004, 305-347.

[5] M. Drmota and R. F. Tichy, Sequences, Discrepancies and Applications, Lecture Notes in Math. 1651, Springer, Berlin, 1997.

[6] B. Gittenberger and J. M. Thuswaldner, Asymptotic normality of b-additive functions on polynomial sequences in the Gaussian number field, J. Number Theory 84 (2000), 317-341.

[7] P. J. Grabner, P. Kirschenhofer, and H. Prodinger, The sum-of-digits function for complex bases, J. London Math. Soc. (2) 57 (1998), 20-40.

[8] K. Gröchenig and A. Haas, Self-similar lattice tilings, J. Fourier Anal. Appl. 1 (1994), 131-170.

[9] L. K. Hua, On exponential sums over an algebraic number field, Canad. J. Math. 3 (1951), 44-51.

[10] K.-H. Indlekofer, I. Kátai, and P. Racskó, Number systems and fractal geometry, in: Probability Theory and Applications, Math. Appl. 80, Kluwer, Dordrecht, 1992, 319-334.

[11] I. Kátai and I. Környei, On number systems in algebraic number fields, Publ. Math. Debrecen 41 (1992), 289-294.

[12] I. Kátai und B. Kovács, Kanonische Zahlensysteme in der Theorie der quadratischen algebraischen Zahlen, Acta Sci. Math. (Szeged) 42 (1980), 99-107.

[13] - - -, Canonical number systems in imaginary quadratic fields, Acta Math. Acad. Sci. Hungar. 37 (1981), 159-164. 
[14] I. Kátai and J. Szabó, Canonical number systems for complex integers, Acta Sci. Math. (Szeged) 37 (1975), 255-260.

[15] D. E. Knuth, The Art of Computer Programming, Vol. 2, 2nd ed., Addison-Wesley, Reading, MA, 1981.

[16] N. M. Korobov, Exponential Sums and their Applications, Math. Appl. (Soviet Ser.) 80, Kluwer, Dordrecht, 1992.

[17] B. Kovács, Canonical number systems in algebraic number fields, Acta Math. Acad. Sci. Hungar. 37 (1981), 405-407.

[18] B. Kovács and A. Pethő, Number systems in integral domains, especially in orders of algebraic number fields, Acta Sci. Math. (Szeged) 55 (1991), 287-299.

[19] M. G. Madritsch, A note on normal numbers in matrix number systems, Math. Pannon. 18 (2007), 219-227.

[20] M. G. Madritsch, J. M. Thuswaldner, and R. F. Tichy, Normality of numbers generated by the values of entire functions, J. Number Theory 128 (2008), 1127-1145.

[21] W. Müller, J. M. Thuswaldner, and R. F. Tichy, Fractal properties of number systems, Period. Math. Hungar. 42 (2001), 51-68.

[22] Y.-N. Nakai and I. Shiokawa, Discrepancy estimates for a class of normal numbers, Acta Arith. 62 (1992), 271-284.

[23] K. Scheicher and J. M. Thuswaldner, Canonical number systems, counting automata and fractals, Math. Proc. Cambridge Philos. Soc. 133 (2002), 163-182.

[24] J. Schiffer, Discrepancy of normal numbers, Acta Arith. 47 (1986), 175-186.

[25] E. C. Titchmarsh, The Theory of the Riemann Zeta-Function, 2nd ed., Oxford Univ. Press, New York, 1986.

[26] I. M. Vinogradov, Selected Works, Springer, Berlin, 1985.

Department for Analysis and Computational Number Theory

Graz University of Technology

Steyrergasse 30

A-8010 Graz, Austria

E-mail: madritsch@tugraz.at

Received on 5.10.2007

and in revised form on 4.8.2008 